Bull. Korean Math. Soc. 52 (2015), No. 5, pp. 1559-1568

http://dx.doi.org/10.4134/BKMS.2015.52.5.1559

\title{
ON RELATIVE CLASS NUMBER AND CONTINUED FRACTIONS
}

\author{
Debopam Chakraborty and Anupam Saikia
}

\begin{abstract}
The relative class number $H_{d}(f)$ of a real quadratic field $K=\mathbb{Q}(\sqrt{m})$ of discriminant $d$ is the ratio of class numbers of $\mathcal{O}_{f}$ and $\mathcal{O}_{K}$, where $\mathcal{O}_{K}$ denotes the ring of integers of $K$ and $\mathcal{O}_{f}$ is the order of conductor $f$ given by $\mathbb{Z}+f \mathcal{O}_{K}$. In a recent paper of A. Furness and E. A. Parker the relative class number of $\mathbb{Q}(\sqrt{m})$ has been investigated using continued fraction in the special case when $\sqrt{m}$ has a diagonal form. Here, we extend their result and show that there exists a conductor $f$ of relative class number 1 when the continued fraction of $\sqrt{m}$ is nondiagonal of period 4 or 5 . We also show that there exist infinitely many real quadratic fields with any power of 2 as relative class number if there are infinitely many Mersenne primes.
\end{abstract}

\section{Introduction}

A real quadratic field $K$ is an extension $\mathbb{Q}(\sqrt{m})=\{a+b \sqrt{m} \mid a, b \in \mathbb{Q}\}$ for some square-free natural number $m$. The discriminant $d$ of $K$ is $m$ if $m \equiv 1$ $\bmod 4$, otherwise $d=4 \mathrm{~m}$. In the former case, the $\operatorname{ring} \mathcal{O}_{K}$ of integers of $K$ is $\left\{a+b \frac{1+\sqrt{m}}{2} \mid a, b \in \mathbb{Z}\right\}$, and in the latter case, $\mathcal{O}_{K}=\{a+b \sqrt{m} \mid a, b \in \mathbb{Z}\}$. By Dirichlet's unit theorem, the units of $\mathcal{O}_{K}$ can be written as $\pm \xi_{m}^{i}(i \in \mathbb{Z})$ where $\xi_{m}$ is called the fundamental unit. The relative class number of $K$ for a conductor $f$ is the ratio $H_{d}(f)$ of the class numbers of the order $\mathcal{O}_{f}=\mathbb{Z}+f \mathcal{O}_{K}$ and $\mathcal{O}_{K}$. It was Dirichlet ([4]) who first obtained several interesting results concerning relative class number of real quadratic fields. Gauss's conjecture on existence of infinitely many real quadratic fields of class number 1 motivated Dirichlet to pose the question whether there are infinitely many real quadratic fields of relative class number 1 (see [2], [4]). The question was successfully addressed in [8], and a characterization in terms of the norm of the fundamental unit was given in [1]. In [6], A. Furness and E. A. Parker studied relative class number of $\mathbb{Q}(\sqrt{m})$ using continued fraction in the special case when $\sqrt{m}$ has a diagonal form [see (3.6)]. The main goal of this paper is to extend their approach and show that there exists a conductor $f$ of relative class number

Received August 14, 2014; Revised January 20, 2015.

2010 Mathematics Subject Classification. Primary 11A55, 11R11, 11R65.

Key words and phrases. relative class number, continued fraction. 
1 when the continued fraction of $\sqrt{m}$ is non-diagonal of period 4 or 5 . The following result of Dirichlet concerning the relative class number will be very useful to us (see $[2]$ ).

Theorem 1.1 (Dirichlet). Let $\theta(f)$ be the smallest positive integer such that $\xi_{m}^{\theta(f)}$ belong to $\mathcal{O}_{f}$ and $\psi(f)=f \prod_{q \mid f}\left(1-\left(\frac{d}{q}\right) \frac{1}{q}\right)$, where $\left(\frac{d}{q}\right)$ denotes the "Kronecker residue symbol" of d modulo a prime $q$. Then the relative class number for conductor $f$ is given by

$$
H_{d}(f)=\frac{\psi(f)}{\theta(f)}
$$

The Kronecker residue symbol $\left(\frac{d}{q}\right)$ is the same as the Legendre symbol when $q$ is an odd prime. For $q=2$ and $d$ odd, $\left(\frac{d}{q}\right)$ is 1 if $d \equiv \pm 1(\bmod 8)$ and -1 if $d \equiv \pm 3(\bmod 8)$. The relative class number is always an integer (see [2]), hence $\theta(f)$ always divides $\psi(f)$. We write the fundamental unit of $\mathcal{O}_{K}$ as

$$
\xi_{m}=\alpha_{0}+\beta_{0} \sqrt{m}, \quad 2 \alpha_{0}, 2 \beta_{0} \in \mathbb{Z} .
$$

Note that $\xi_{m}^{3} \in \mathbb{Z}[\sqrt{m}]$ and when $m \not \equiv 5(\bmod 8), \alpha_{0}$ and $\beta_{0}$ are integers (see [7]). For the rest of the paper, we use the following notation:

$$
\tilde{\beta_{0}}=\beta_{0}, \tilde{\alpha_{0}}=\alpha_{0} \text { if } \xi_{m} \in \mathbb{Z}[\sqrt{m}], \quad \tilde{\beta}_{0}=2 \beta_{0}, \tilde{\alpha_{0}}=2 \alpha_{0} \text { if } \xi_{m} \notin \mathbb{Z}[\sqrt{m}] .
$$

\section{Continued fraction approach}

In [6], the authors showed that the existence of relative class number 1 can be related to the continued fraction of $\sqrt{m}$. Their results were for those $m$ for which $\sqrt{m}$ has a continued fraction of diagonal form (see (3.6)). We are now going to extend that approach and prove that whenever $\sqrt{m}$ is represented by a continued fraction of period 4 or 5 , there exists a conductor $f$ with relative class number 1 . When $m$ is a square-free positive integer, it is well-known (e.g., see [9]) that the continued fraction of $\sqrt{m}$ is periodic of the form

$$
n+\frac{1}{a_{1}+\frac{1}{\cdots+\frac{1}{a_{r}+\frac{1}{2 n+\frac{1}{a_{1}+\cdots}}}}}, \quad \text { where } n=\lfloor\sqrt{m}\rfloor \text {, and } a_{i}=a_{r+1-i} \text {. }
$$

It is standard to denote it as

$$
\sqrt{m}=\left\langle n, \overline{a_{1}, a_{2}, \ldots, a_{r}, 2 n}\right\rangle .
$$

The main result of this paper is the following theorem.

Theorem 2.1. Let $m$ be a square-free positive integer such that $\sqrt{m}$ is represented by a continued fraction of period 4 or 5 . Then there exists a prime divisor $p$ of $m$ such that the relative class number for $p$ is 1 . In other words, $H_{d}(p)=1$ where $d$ is the discriminant of the number field $\mathbb{Q}(\sqrt{m})$. 
In order to prove the above theorem, we will use Dirichlet's formula (1.1) which involves the fundamental unit $\xi_{m}$ of the real quadratic field $\mathbb{Q}(\sqrt{m})$. It is well known that $\xi_{m}$ is closely related to the continued fraction for $\sqrt{m}$. Hence we will first look into properties of the continued fraction for $\sqrt{m}$.

\section{Useful properties of the continued fraction of $\sqrt{m}$}

It is convenient for us to write the continued fraction of $\sqrt{m}$ in $(2.2)$ as

$$
\sqrt{m}=\langle n, x\rangle=n+x^{-1}, \text { where } x=\left\langle\overline{a_{1}, a_{2}, \ldots, a_{r}, 2 n}\right\rangle .
$$

The $i$-th convergent of the continued fraction of $x$ is defined as

$$
\frac{h_{i}}{k_{i}}=a_{1}+\frac{1}{a_{2}+\frac{1}{\cdots+\frac{1}{a_{i+1}}}} .
$$

It is easy to verify that the following recurrence relations are satisfied by $h_{i}$ and $k_{i}$ :

$$
\begin{aligned}
& h_{0}=a_{1}, \quad k_{0}=1, \quad h_{1}=1+a_{1} a_{2}, \quad k_{1}=a_{2}, \\
& h_{i}=a_{i} h_{i-1}+h_{i-2}, \quad k_{i}=a_{i} k_{i-1}+k_{i-2}, \\
& h_{i} k_{i-1}-h_{i-1} k_{i}=(-1)^{i-1} .
\end{aligned}
$$

Conventionally, the explicit expressions in terms of the $a_{i}$ s for the partial quotients $h_{i}$ and $k_{i}$ are denoted by

$$
h_{i-1}=\left[a_{1}, a_{2}, a_{3}, \ldots, a_{i}\right], \quad k_{i-1}=\left[a_{2}, a_{3}, \ldots, a_{i}\right],
$$

(e.g. see [3]),

$$
\begin{aligned}
& h_{2}=\left[a_{1}, a_{2}, a_{3}\right]=a_{1} a_{2} a_{3}+a_{1}+a_{3}, \\
& h_{3}=\left[a_{1}, a_{2}, a_{3}, a_{4}\right]=a_{1} a_{2} a_{3} a_{4}+a_{3} a_{4}+a_{1} a_{4}+a_{1} a_{2}+1 .
\end{aligned}
$$

The Euler's rule (see [3]) tells us that the expressions for partial quotients in terms of the $a_{i}$ s are unchanged when we take the $a_{i}$ s in the reverse order, i.e.,

$$
\left[a_{1}, a_{2}, a_{3}, \ldots, a_{k}\right]=\left[a_{k}, a_{k-1}, \ldots, a_{2}, a_{1}\right] .
$$

We first prove the following lemma, which completely generalizes an analogue in [6] for $\sqrt{m}$ with a continued fraction of diagonal form, i.e.,

$$
\sqrt{m}=\langle n, \overline{a, \ldots, a, 2 n}\rangle .
$$

Lemma 3.1. Let $\sqrt{m}=\left\langle n, \overline{a_{1}, a_{2}, \ldots, a_{r}, 2 n}\right\rangle$ and let $\frac{h_{i}}{k_{i}}$ be the $i$-th convergent of the purely periodic continued fraction $x=\left\langle\overline{a_{1}, a_{2}, \ldots, a_{r}, 2 n}\right\rangle$. Then we have

$$
m=n^{2}+\frac{k_{r}}{h_{r-1}} .
$$

In particular, $h_{r-1}$ divides $k_{r}$. 
Proof. Note that $\sqrt{m}=\langle n, x\rangle=n+x^{-1}$. Now, $x$ can also be written as

$$
x=\left\langle a_{1}, a_{2}, \ldots, a_{r}, 2 n, \overline{a_{1}, a_{2}, \ldots, a_{r}, 2 n}\right\rangle=\left\langle a_{1}, a_{2}, \ldots, a_{r}, 2 n, x\right\rangle .
$$

Therefore, the $(r+1)$-th convergent of $\left\langle a_{1}, a_{2}, \ldots, a_{r}, 2 n, x\right\rangle$ equals $x$. By the recurrence relations given in (3.2), the $(r+1)$-th convergent of $\left\langle a_{1}, a_{2}, \ldots, a_{r}, 2 n\right.$, $x\rangle$ is given by

$$
\frac{x h_{r}+h_{r-1}}{x k_{r}+k_{r-1}}
$$

As a consequence, we have

$$
x=\frac{x h_{r}+h_{r-1}}{x k_{r}+k_{r-1}},
$$

and we obtain the following quadratic equation for $x^{-1}$ :

$$
x^{-2}\left(h_{r-1}\right)+x^{-1}\left(h_{r}-k_{r-1}\right)-k_{r}=0 .
$$

Using Euler's rule (3.5) for the partial quotients $h_{i}$ and $k_{i}$ of $x$ and then using the fact that $a_{i}=a_{r+1-i}$ from (2.1), we obtain

$$
k_{r-1}=\left[a_{2}, a_{3}, \ldots, a_{r}\right]=\left[a_{r}, a_{r-1}, \ldots, a_{2}\right]=\left[a_{1}, a_{2}, \ldots, a_{r-1}\right]=h_{r-2} .
$$

On substituting in the quadratic equation (3.7), we obtain

$$
x^{-2}\left(h_{r-1}\right)+x^{-1}\left(h_{r}-h_{r-2}\right)-k_{r}=0 .
$$

In view of the recurrence relation (3.2), we find that $h_{r}=2 n h_{r-1}+h_{r-2}$. Therefore, the quadratic equation for $x^{-1}$ simplifies to

$$
x^{-2}\left(h_{r-1}\right)+x\left(2 n h_{r-1}\right)-k_{r}=0,
$$

and we obtain its solution

$$
x^{-1}=-n+\sqrt{n^{2}+\frac{k_{r}}{h_{r-1}}} \text { as } x>0 .
$$

Thus we find that $\sqrt{m}=n+x^{-1}=\sqrt{n^{2}+\frac{k_{r}}{h_{r-1}}}$ and hence

$$
m=n^{2}+\frac{k_{r}}{h_{r-1}} .
$$

As $m$ is an integer, it now follows trivially that $k_{r}$ divides $h_{r-1}$.

Next, we obtain a bound on the coefficients appearing in the continued fraction of $\sqrt{m}$. We will use this bound in the next section.

Proposition 3.2. Let $\sqrt{m}=\langle n ; \overline{a, b, \ldots}\rangle$ be a continued fraction of period at least 3. Then $a b<2 n$. 
Proof. Let $[\sqrt{m}]=n$ so that $m=n^{2}+t$ where $t$ must be an integer satisfying $t \leq 2 n$. Then

$$
\sqrt{m}=n+\sqrt{m}-n=n+\frac{1}{\frac{\sqrt{m}+n}{m-n^{2}}}=n+\frac{1}{\frac{2 n+(\sqrt{m}-n)}{t}}, \quad 0<\sqrt{m}-n<1 .
$$

Now, the next coefficient $a$ in the continued fraction of $\sqrt{m}$ is given by

$$
2 n=t a+r_{1}, \quad 0<r_{1}<t .
$$

Note that $r_{1}=0$ would imply that $\sqrt{m}$ has continued fraction $\langle n ; \overline{a, 2 n}\rangle$ of period 2. Now,

$$
\sqrt{m}=n+\frac{1}{\frac{t a+\left(\sqrt{m}-\left(n-r_{1}\right)\right)}{t}}=n+\frac{1}{a+\frac{1}{\frac{t\left(\sqrt{m}+\left(n-r_{1}\right)\right)}{m-\left(n-r_{1}\right)^{2}}}} .
$$

Now, the last denominator is

$$
m-\left(n-r_{1}\right)^{2}=\left(m-n^{2}\right)+2 n r_{1}-r_{1}^{2}=t+\left(t a+r_{1}\right) r_{1}-r_{1}^{2}=t\left(1+a r_{1}\right),
$$

and the numerator is

$$
t\left(\sqrt{m}+\left(n-r_{1}\right)\right)=t\left(2 n-r_{1}+\sqrt{m}-n\right) .
$$

Now, the next coefficient $b$ in the continued fraction of $\sqrt{m}$ is given by

$$
2 n-r_{1}=\left(1+a r_{1}\right) b+r_{2}, \quad 0 \leq r_{2}<1+a r_{1} .
$$

As $r_{1} \geq 1$, we deduce from the last equality that $2 n>a b$.

\section{Proof of the theorem}

We are now going to use the properties of the continued fraction of $\sqrt{m}$ deduced in $\S 3$ to prove theorem 2.1. It is well known (see [7]) that $n h_{r-1}+$ $h_{r-2}+h_{r-1} \sqrt{m}$ is the fundamental unit $\xi_{m}$ of $\mathbb{Q}(\sqrt{m})$ except in the case $m \equiv 5$ $(\bmod 8)$, when it equals $\xi_{m}^{3}$. Therefore, $h_{r-1}$ is always a multiple of $\tilde{\beta}_{0}$ (see $(1.2))$.

If we can show that $m$ does not divide $h_{r-1}$ then there will be a prime factor $p$ of $m$ which does not divide $h_{r-1}$ as $m$ is square-free. Such a prime $p$ will not divide $\tilde{\beta}_{0}$ as well. For such a prime $p$, it is obvious that $\theta(p)$ will be $p$ and $\psi(p)$ will also be $p$ in Dirichlet's formula (1.1). Therefore, such a prime $p$ will be a conductor of relative class number 1 . In the first subsection below, we will prove that if the continued fraction for $\sqrt{m}$ has period 4 then $m$ does not divide $h_{r-1}$, which ensures the existence of a prime conductor $p$ of relative class number 1 . In the second subsection, we will do the same when the continued fraction for $\sqrt{m}$ has period 5 . 


\subsection{When $\sqrt{m}$ has a continued fraction of period 4}

Suppose $\sqrt{m}$ has a continued fraction of period $4(r=3$ in Lemma 3.1). As outlined in $\S 3(2.1)$, the continued fraction of $\sqrt{m}$ is necessarily of the form $\sqrt{m}=\langle n, \overline{a, b, a, 2 n}\rangle$. It is clear from the preceding paragraph that theorem 2.1 in this case reduces to the following proposition.

Proposition 4.1. If $\sqrt{m}=\langle n, \overline{a, b, a, 2 n}\rangle$, then $m$ does not divide $h_{2}$.

Proof. We first consider the case when $b>3$. It is enough to show that $h_{2} \leq n^{2}$ where $n=\lfloor\sqrt{m}\rfloor$ as in (2.1). By (3.3) and (3.4) we have

$$
\begin{aligned}
h_{2} & =[a, b, a]=a^{2} b+2 a, \\
h_{1} & =[a, b]=a b+1=k_{2}, \\
k_{1} & =[b]=b .
\end{aligned}
$$

As $h_{2} k_{1}-k_{2} h_{1}=-1$ (by $\left.(3.2)\right)$, we deduce that

$$
k_{2}^{2} \equiv 1\left(\bmod h_{2}\right) \text {. }
$$

By Lemma 3.1, $h_{2}$ divides $k_{3}$. Using the recurrence relation (3.2) for $k_{3}$, we find that

$$
2 n k_{2}+k_{1}=k_{3} \equiv 0\left(\bmod h_{2}\right) .
$$

Multiplying both sides by $k_{2}$ and using the relation (4.2), we obtain

$$
2 n \equiv 2 n k_{2}^{2} \equiv-k_{2} k_{1} \equiv-a b^{2}-b\left(\bmod h_{2}\right) .
$$

Multiplying both sides by $a$, and using $h_{2}=a^{2} b+2 a$ from (4.1), we obtain

$$
2 n a \equiv-a^{2} b^{2}-a b \equiv a b\left(\bmod h_{2}\right) .
$$

By proposition 3.2 , we have $2 n>b$ and consequently there is a positive integer $l$ such that

$$
2 n a=l\left(h_{2}\right)+a b=l a(a b+2)+a b .
$$

Consequently, $2 n \geq a b+b+2$ and

$4 n^{2}>a^{2} b^{2}+4 a b+4+2 a b^{2}+4 b+b^{2}>a^{2} b^{2}+4 a b \geq 4\left(a^{2} b+2 a\right)=4 h_{2} \quad($ as $b>3)$.

Therefore, $m>n^{2}>h_{2}$ and in particular $m$ can not divide $h_{2}$ when $b>3$.

For $b=1,2$ or 3 , we use the corresponding expression for $h_{2}$ in terms of $a$, and then find a suitable upper bound for $a$ in terms of $n$. As noted in (3.9), the coefficient $a$ in the continued fraction for $\sqrt{m}$ is given by

(4.3) $2 n=t a+r_{1}$, where $t=m-n^{2}$, and $r_{1}>0$ (as the period of $\sqrt{m}$ is 4$)$.

But $t$ is at least 2 as otherwise,

$$
m=n^{2}+t=n^{2}+1 \Rightarrow \sqrt{m}=\langle n, \overline{2 n}\rangle \text { (of period 1). }
$$

Consequently $2 a<2 n$ from (4.3) and $a \leq n-1$. Observe also that $n>1$ as $\sqrt{m}=\sqrt{2}$ or $\sqrt{3}$ is of period strictly less than 4 .

For $b=1$, we now have

$$
h_{2}=[a, 1, a]=a^{2}+2 \leq(n-1)^{2}+2 \leq n^{2}<m \text { as } a \leq n-1 .
$$


For $b=2$, we have

$$
h_{2}=[a, 2, a]=2 a^{2}+2 a \leq 2(n-1)^{2}+2(n-1) \leq 2 n^{2}<2 m .
$$

If $m$ divides $h_{2}$, then that would mean $h_{2}=m$. But then $m=h_{2}=2 a(a+1)$ will not be square free as 4 divides $2 a(a+1)$.

For $b=3$, we have $h_{2}=3 a^{2}+2 a$ and we need a sharper upper bound for $a$ than just $n-1$. By (4.3), it amounts to finding a sharper lower bound for $t$. We have

$$
t=\frac{2 n-r_{1}}{a}>\frac{2 n-\left(r_{1}+r_{2}\right)}{1+a r_{1}}=b,
$$

where the last equality above is due to (3.10). Thus $t \geq 4$ and by (4.3)

$$
a=\frac{2 n-r_{1}}{t} \leq \frac{2 n-1}{4}=\frac{n}{2}-\frac{1}{4} \Rightarrow a \leq \frac{n-1}{2}
$$

as $a$ is an integer. Finally,

$$
h_{2}=3 a^{2}+2 a \leq \frac{3}{4}(n-1)^{2}+(n-1) \leq n^{2}<m .
$$

\subsection{When $\sqrt{m}$ has a continued fraction of period 5}

Suppose $\sqrt{m}$ has a continued fraction of period $5(r=4$ in Lemma 3.1). As outlined in $\S 3(2.1)$, it is clear that the continued fraction of $\sqrt{m}$ is necessarily of the form $\sqrt{m}=\langle n, \overline{a, b, b, a, 2 n}\rangle$. By the paragraph preceding $\S 4$.1, we need only to show that $m$ does not divide $h_{3}$. Therefore, Theorem 2.1 in this case reduces to the following proposition.

Proposition 4.2. If $\sqrt{m}=\langle n, \overline{a, b, b, a, 2 n}\rangle$, then $m>h_{3}$.

Proof. Recall that $\sqrt{m}=\sqrt{n^{2}+\frac{k_{r}}{h_{r-1}}}$ when $\sqrt{m}=\left\langle n, \overline{a_{1}, a_{2}, \ldots, a_{r}, 2 n}\right\rangle$ where, $a_{i}=a_{r+1-i}$, and $\frac{h_{i}}{k_{i}}$ denotes the $i$-th convergent. Here, $r=4$. By (3.3) and (3.4), we have

$$
\begin{aligned}
h_{3} & =[a, b, b, a]=(a b+1)^{2}+a^{2}=\left(a^{2} b^{2}+a^{2}+a b\right)+(a b+1) \\
k_{3} & =[b, b, a]=a b^{2}+a+b \\
h_{2} & =[a, b, b]=k_{3} \\
k_{2} & =[b, b]=b^{2}+1
\end{aligned}
$$

and we obtain

$$
k_{3}^{2} \equiv(-1)^{3}=-1\left(\bmod h_{3}\right) \quad(\text { as in }(4.2)) .
$$

By Lemma $3.1 h_{2}$ divides $k_{3}$. Using the recurrence relation $(3.2)$ for $k_{2}$, we find that

$$
2 n k_{3}+k_{2}=k_{4} \equiv 0\left(\bmod h_{3}\right)
$$

By (4.5), we obtain

$$
2 n \equiv-2 n k_{3}^{2} \equiv k_{3} k_{2} \equiv\left(a b^{2}+a+b\right)\left(b^{2}+1\right)\left(\bmod h_{3}\right) .
$$


Multiplying both sides by $a$ and then using the expression for $h_{3}$ in (4.4) we obtain

$$
2 n a \equiv\left(a^{2} b^{2}+a^{2}+a b\right)\left(b^{2}+1\right) \equiv-(a b+1)\left(b^{2}+1\right)\left(\bmod h_{3}\right) .
$$

Multiplying both sides by $(1+a b)$ then using the expression for $h_{3}$ in (4.4) we get

$$
2 n a(a b+1) \equiv-(a b+1)^{2}\left(b^{2}+1\right) \equiv a^{2}\left(b^{2}+1\right)\left(\bmod h_{3}\right),
$$

and consequently,

$$
2 n(a b+1) \equiv a\left(b^{2}+1\right)\left(\bmod h_{3}\right) \quad\left(\operatorname{as} \operatorname{gcd}\left(a, h_{3}\right)=1 \text { from }(4.4)\right) .
$$

Therefore, there is an integer $l$ such that

$$
2 n(a b+1)=l h_{3}+a\left(b^{2}+1\right)=l\left((a b+1)^{2}+a^{2}\right)+a\left(b^{2}+1\right) .
$$

By Proposition 3.2, $2 n>a b$ and hence $l$ must be a positive integer. We now claim that $l$ must be even from parity considerations in the last equality. When $a$ is even or $a, b$ both are odd, it is clear from (4.1) that $h_{3}$ is odd and then it follows from (4.6) that $l$ must be even. If $a$ is odd and $b$ is even, $h_{3}$ is even but $a\left(b^{2}+1\right)$ is odd which is ruled out by parity consideration in the first equality of (4.6). Putting $l \geq 2$ in (4.6), we obtain

$$
2 n \geq 2(a b+1)+\frac{2 a^{2}+a\left(b^{2}+1\right)}{a b+1},
$$

and by squaring both sides, we get

$$
4 n^{2} \geq 4(a b+1)^{2}+4\left(2 a^{2}+a\left(b^{2}+1\right)\right)+\left(\frac{2 a^{2}+a\left(b^{2}+1\right)}{a b+1}\right)^{2} .
$$

It follows that

$$
4 n^{2}>4(a b+1)^{2}+4 a^{2}=4 \cdot h_{3}
$$

and as a result

$$
m>n^{2}>h_{3}
$$

The following result also comes out in the proof.

Corollary 4.3. There does not exist square free positive integer $m$ such that $\sqrt{m}=\langle n, \overline{a, b, b, a, 2 n}\rangle$ and $a$ is odd and $b$ is even.

\section{Certain interesting implications}

We conclude with two interesting observations that follow from our results. The first observation is that we can easily construct an infinite family real quadratic fields of class number 1 . The second observation is that there are infinitely many real quadratic fields with any given power of 2 as relative class number provided there are infinitely many Mersenne primes. We explain these two observations below.

If $m$ is a square-free integer of the form $n^{2}+n$ then it follows that $\sqrt{m}=$ $\langle n, \overline{2,2 n}\rangle$. Here, $h_{r-1}=2 \Rightarrow \theta(2)=1$ and $\psi(2)=2$ as 2 always divides $n^{2}+n$. So, $H_{d}(2)$ is 2 . As $n^{2}+n$ can not be power of 2 , it has an odd prime divisor $p$ 
dividing $m$. As $p$ does not divide $2=h_{r-1}$, we must have $H_{d}(p)=1$. Note that there exist infinitely many square-free consecutive integers $n$ and $(n+1)$ : if $N$ is a sufficiently large natural number and we sieve out all the integers between $2 k N+1$ to $(2 k+2) N$ which are divisible by squares of prime, we will be left more than $N$ square-free integers as

$$
\sum \frac{1}{p^{2}}<0.4522474200 \cdots<\frac{1}{2}
$$

(see [5]) and hence at least two of those square-free integers will be consecutive. Hence there exist infinitely many square free positive integers of the from $m=n^{2}+n$ giving relative class number 1 for any odd prime divisor of $m$ as conductor.

Now suppose $m>6$ is an integer which is twice a Mersenne prime. In other words, let $m=2\left(2^{p}-1\right)$ where $p=2 k+1$ is a prime. Then, $m$ is square free and we have

$m=\left(2^{k}\right)^{2}-2=(n+1)^{2}-2, \quad \sqrt{m}=\langle n, \overline{1, n-1,1,2 n}\rangle, \quad h_{r-1}=n+1=2^{k}$. As $\xi_{m} \in 2^{i} \mathcal{O}_{K}$ for all $0 \leq i \leq k, \theta\left(2^{i}\right)=1$. Now,

$$
\psi\left(2^{i}\right)=2^{i}\left(1-\left(\frac{d}{p}\right) \frac{1}{p}\right)\left(1-\left(\frac{d}{2}\right) \frac{1}{2}\right)=2^{i} .
$$

Therefore $H_{d}\left(2^{i}\right)=2^{i}$. If we assume that the number of Mersenne primes is infinite, there will be infinitely many primes of the form $2^{2 k-1}-1$, and hence we can demonstrate infinitely many real quadratic fields with any power of 2 as relative class number.

\section{References}

[1] D. Chakraborty and A. Saikia, Another look at real quadratic fields of relative class number 1, Acta Arith. 163 (2014), no. 4, 371-378.

[2] H. Cohn, A numerical study of the relative class numbers of real quadratic integral domains, Math. Comp. 16 (1962), 127-140.

[3] H. Davenport, Higher Arithmetic, Cambridge University Press, 2008.

[4] P. G. L. Dirichlet, Sur une propriété des formes quadratiques à déterminant positif, Math. Pures Appl. Ser II 1 (1856), 76-79.

[5] S. R. Finch, Mathematical Constants, Cambridge University Press, 2003.

[6] A. Furness and E. A. Parker, On Dirichlet's conjecture on relative class number one, J. Number Theory 132 (2012), no. 7, 1398-1403.

[7] R. Mollin, Quadratics, CRC Press, 1996.

[8] — Proof of relative class number one for almost all real quadratic fields and a counterexample for the rest, Gen. Math. Notes 17 (2013), no. 2, 81-90.

[9] I. Niven, H. S. Zuckerman, and H. L. Montgomery, An Introduction to the Theory of Numbers, John Wiley and Sons Inc., U.K., Fifth Edition, 2008.

Debopam Chakraborty

Department of Mathematics

Indian Institute of TECHNOLOGY

GuWAhati-781039, Assam, India

E-mail address: c.debopam@iitg.ernet.in 
ANUPAM SAIKIA

Department of Mathematics

Indian Institute of TeChNOLOGY

Guwahati-781039, Assam, India

E-mail address: a.saikia@ittg.ernet.in 